\title{
Multiscale analysis of pattern formation via intercellular signalling
}

\author{
O'Dea, R.D. \& King, J.R.* \\ ${ }^{*}$ Centre for Mathematical Medicine and Biology, \\ School of Mathematical Sciences, \\ University of Nottingham, University Park, \\ Nottingham, NG7 2RD, UK
}

June 10, 2015

\begin{abstract}
Lateral inhibition, a juxtacrine signalling mechanism by which a cell adopting a particular fate inhibits neighbouring cells from doing likewise, has been shown to be a robust mechanism for the formation of fine-grained spatial patterns (in which adjacent cells in developing tissues diverge to achieve contrasting states of differentiation), provided that there is sufficiently strong feedback. The fine-grained nature of these patterns poses problems for analysis via traditional continuum methods since these require that significant variation takes place only over lengthscales much larger than an individual cell and such systems have therefore been investigated primarily using discrete methods. Here, however, we apply a multiscale method to derive systematically a continuum model from the discrete Delta-Notch signalling model of Collier et al. (Pattern formation by lateral inhibition with feedback: a mathematical model of Delta-Notch intercellular signalling, J. Theor. Biol., 183, 1996, 429-446) under particular assumptions on the parameters, which we use to analyse the generation of fine-grained patterns. We show that, on the macroscale, the contact-dependent juxtacrine signalling interaction manifests itself as linear diffusion, motivating the use of reaction-diffusion-based models for such cell-signalling systems. We also analyse the travelling-wave behaviour of our system, obtaining good quantitative agreement with the discrete system.
\end{abstract}

\section{Introduction}

The development of spatial organisation is fundamental to the construction of multicellular organisms: starting from a homogeneous initial state, a collection of cells must have the ability to arrange themselves in a certain configuration to enable the creation of specialised body parts. This spatial organisation impacts upon cell differentiation and the determination of cell fate (i.e. the adoption of a particular programme of gene activation). Cell fates are regulated by a variety of mechanisms, typically mediated by the production and transport of certain signalling molecules that bind to specific sites on the cell membrane. In many developing tissues fine-grained patterning is generated, in which neighbouring cells diverge to achieve different cell fates. Lateral inhibition, a juxtacrine signalling mechanism (in which the signalling molecule is anchored in the cell membrane and acts only on neighbouring cells) is one process by which such patterns are generated and is well-documented in flies, fishes, worms and invertebrates, and has been observed in the developing nervous system in 
particular (Collier et al., 1996; Haddon, 1998; Appel et al., 2001). In all of these systems the transmembrane proteins Notch and Delta (or their homologues) have been identified as mediators of the interaction. Within the context of regenerative medicine, Delta-Notch signalling has been shown to regulate cell fate in stem-cell clusters, with high Delta expression preventing differentiation and improving cohesiveness (Lowell et al., 2000), maintaining regenerative potential in muscle cells (Conboy et al., 2003) and regulating stem cell numbers both in vitro and in vivo (Androutsellis-Theotokis et al., 2006). Many other ligand-receptor interactions and subsequent intracellular signalling cascades have been identified as cell fate determination mechanisms and subsequently studied in the mathematical biology literature; examples include the binding of cyclic AMP to Dictyostelium cells (Shaapa et al., 1986; Dallon \& Othmer, 1997), Transforming and Epidermal Growth Factor binding in keratinocytes (Coffey et al., 1987; Ebner \& Derynck, 1991; Wiley et al., 2003) and Wnt binding in a variety of stem cell types (Nusse, 2008; Mirams et al., 2010). However, in this paper, we concentrate on the well-understood Delta-Notch juxtacrine signalling system, which provides an ideal model system for the type of analysis which we seek to pursue and, after Collier et al. (1996), we neglect any intracellular detail.

Due to its importance in the understanding of tissue formation, the development of spatial patterning in response to biologically-relevant mechanisms has been widely studied. Inspired by the study of Turing (1952), who showed that reaction and diffusion of chemicals can produce spatial patterning in chemical concentration that consequently determines cell fate, reaction-diffusion systems have been employed in modelling a variety of biological systems. For instance, Painter et al. (1999) showed that patterned solutions of striking similarity to animal coat patterns can be generated by such a model. An alternative mechanism was proposed by Wolpert (1969) in which the level of a morphogen (whose gradient is established via diffusion from a fixed source) determines cell fate; Lander et al. (2002) have provided evidence that diffusive transport and ligand-receptor interaction is able to set up appropriate morphogen gradients. Most such studies employ a continuum approach (though it is noteworthy that Turing (1952) exploited both discrete and continuous formulations); however, a number of relatively recent studies have employed discrete models to analyse signalling processes at the cell scale. Collier et al. (1996) presented the first model of juxtacrine signalling, considering the activity of a protein, Delta, and its receptor, Notch, showing that lateral inhibition is able to generate fine-grained spatial patterns. The model was formulated in terms of ordinary differential equations (ODEs) representing Delta-Notch signalling activity in each cell; Webb \& Owen (2004b) extended this model, considering the dynamics of ligand and free and bound receptors in systems of varying geometry (strings and square or hexagonal arrays), showing that lateral inhibition can produce patterns with a lengthscale of many cell diameters. In the limit of slow ligand-receptor binding, this model is equivalent to that of Collier et al. (1996). Webb \& Owen (2004a) investigated the effects of cell polarity, demonstrating that coherent arrays of polarised cells (analogous to the polarity of bristles and hairs in Drosophila) can be produced.

The analysis of discrete models can be highly numerical in nature, and realistic simulations demand large numbers of cells; this is presumably in part why much research has concentrated until recently on continuous reaction-diffusion models. However, as well as exchanging matter (by diffusion and active transport) cells interact by exchanging signals via specific signalling mechanisms. Indeed, Plahte (2001) and Plahte \& Øyehaug (2007) remark that it is unclear that diffusion is a meaningful approximation for these interactions, especially when considering juxtacrine signalling. Nevertheless, Roussel \& Roussel (2004) demonstrated that such a mechanism may be represented by a diffusion process within a 
continuum formulation, provided that the signalling-molecule concentration varies slowly in comparison to the length of a cell - this result, however, is not applicable in the current context in which neighbouring cells can exhibit very different behaviour. In this paper, we employ a multiscale technique to derive a continuum model of juxtacrine intercellular signalling (based on the Delta-Notch model of Collier et al. (1996)) which is nevertheless able to capture fine-grained patterns with significant variation in signalling molecule concentration between adjacent cells. The resulting continuum model allows representation of the generation of microscale patterns in a tissue in response to macroscale variation in cell signalling (e.g. that induced by tissue-level chemical or physical stimulation). Our reduced model takes the form of a semilinear partial differential equation and is therefore amenable to well-established methods of both analytic and numerical analysis and we demonstrate that, on the macroscale, the interaction between adjacent cells does in fact manifest itself as linear diffusion, despite the short-range variation in signal concentration. We employ this model to investigate the development of fine-grained patterns in response to spatial variation of the strength of the nearest-neighbour feedback mechanism. Comparison with simulations of the discrete system shows that, in the appropriate parameter regimes, the continuum model faithfully reproduces the behaviour of the underlying discrete system. Quantitative comparison between the continuum and discrete models is made by analysing their travelling-wave behaviour, in particular.

The method of multiple-scale expansions for partial differential equations is well-developed (see, for example, Kevorkian \& Cole (1996)) and widely used to derive models for a variety of physical and biological problems. Representative examples include Burridge \& Keller (1981), in which a two-scale homogenisation technique was employed to derive the equations of linear poroelasticity for a porous material, while, in a biological context, Goel et al. (2006) have derived a diffusion-type equation for calcium dynamics within the cell cytoplasm, which was viewed as constituting the endoplasmic reticulum (ER) surrounded by cytosol: the homogenised diffusion coefficients were found to be dependent upon the underlying geometry of the ER, and an example calculation for a specific geometry was given. Such techniques have also been employed to derive continuum representations of collective movement of adherent cells by, for instance, Turner et al. (2004) and Fozard et al. (2009). We remark that in many studies which exploit a homogenisation technique to derive a continuum model from an underlying discontinuous system, local periodicity is in effect imposed by the geometry of the physical system considered, such as the repeating ER/cytosol pattern in Goel et al. (2006) or the individual material pore in Burridge \& Keller (1981). In contrast, in the Delta-Notch signalling system considered here, periodicity is an emergent property of the nearest-neighbour interactions between identical cells rather than being enforced a priori. This periodicity can be exploited by judicious choice of variables to obviate the complications that arise in many homogenisation problems.

The structure of this paper is as follows. In $\S 2$, a short summary of the juxtacrine signalling model of Collier et al. (1996) is given and its pattern-forming behaviour analysed: the patterning bifurcation structure in small populations of cells is demonstrated and the range of patterns exhibited by the model discussed. A continuum model for Delta-Notch signalling in a line of cells based upon this underlying discrete system is then derived in $\S 3$. In $\S 4$, numerical solutions to the continuum equations are presented and compared to simulations of the discrete system; pattern-generating travelling waves are considered, as well as competition between stable patterns. In Appendix A, an indication of the (surprisingly wide) range of parameter values for which our continuum limit applies is provided via comparison of the speed of a linearly-selected travelling wave in the continuous and 
discrete systems. In $\S 5$, a two-dimensional continuum model is derived by considering an array of square cells and comparisons to the underlying discrete system are again made. A discussion, together with suggestions for further work, is given in $\S 6$. The analysis is generalised somewhat in Appendix B, illustrating how the range of applicability of our continuum approach may be significantly extended (albeit in a rather artificial context), with a nonlinear diffusion term resulting in the relevant limit.

\section{Pattern formation in a discrete Delta-Notch intercellular signalling model}

\subsection{Formulation}

In Collier et al. (1996), the activity of a signalling protein, Delta, and its receptor, Notch, is studied. This interaction is known to be important in early animal development. The crucial aspect of the feedback loop considered is that high Delta expression in a cell downregulates Delta expression in its neighbours via the Notch receptor. This mechanism, known as lateral inhibition, is a fundamental cell-fate control mechanism (Mitsiadis et al., 1999), creating fine-grained patterns in developing tissue that determine subsequent cell development. The model is formulated in terms of Delta and Notch "activity" (there is some ambiguity in how this is to be interpreted; see Collier et al. (1996) for a discussion of possible interpretations) and the key postulate is that the level of activated Notch in a cell determines its fate: low levels lead to the adoption of the default (primary) fate, whilst high levels relegate the cell to the secondary fate. In the specific case of nervous tissue in Drosphila, the primary fate corresponds to the adoption of a neural phenotype, the secondary fate being the maintenance of the epidermal phenotype (Lehmann et al., 1983; Campos-Ortega, 1993). The details of the signalling pathways are neglected for simplicity. Cell division is neglected since cell cycle times significantly exceed the timescale of Delta-Notch-mediated cell-fate determination (Hartenstein \& Posakony, 1990). We return to the idealised model of Collier et al. (1996) in place of the more complex signalling models discussed in $\S 1$ since our intention here is in large part to investigate the applicability of multiscale methodologies, rather than to explore in detail the biological implications. Our multiscale approach may be applied mutatis mutandis to more involved signalling models and other cellular geometries; such investigations will be presented elsewhere..

The model comprises a pair of ODEs that govern the evolution of the levels of Delta $\left(d_{j}(t)\right)$ and Notch $\left(n_{j}(t)\right)$ activity in each cell $(j)$ over time $(t)$. In dimensionless terms these are (Collier et al., 1996):

$$
\begin{aligned}
& \dot{d}_{j}=\lambda\left(g\left(n_{j}\right)-d_{j}\right), \\
& \dot{n}_{j}=f\left(\bar{d}_{j}\right)-n_{j},
\end{aligned}
$$

where dots denote differentiation with respect to time and $\lambda$ is the ratio of the decay rates of Delta and Notch activity. In (1), $f\left(\bar{d}_{j}\right)$ and $g\left(n_{j}\right)$ are feedback functions representing the coupling between adjacent cells and the inhibitory effect of Delta-Notch binding, respectively; $\bar{d}_{j}$ denotes the mean level of Delta activity in the cells surrounding cell $j$, i.e.

$$
\bar{d}_{j}=\frac{1}{N} \sum_{i} d_{i}
$$


where $i$ runs over all $N$ nearest neighbours of $j$. In a one-dimensional string of cells (2) therefore reduces to $\bar{d}_{j}=\left(d_{j+1}+d_{j-1}\right) / 2$. To model the lateral inhibition exerted by one cell upon another, the feedback functions employed in Collier et al. (1996) are specified as follows:

$$
f(\sigma)=\frac{\sigma^{k}}{a+\sigma^{k}}, \quad g(\sigma)=\frac{1}{1+b \sigma^{h}},
$$

key aspects of these expressions being that $f$ is an increasing saturating function and $g$ is decreasing (to model the inhibitory effect of the bound Delta-Notch complexes upon Delta production). The positive parameters $k, h, a$ and $b$ determine the feedback strength. The value of the exponent $k$ expresses whether Delta-Notch binding is monovalent $(k=1)$ or cooperative $(k \geqslant 2)$; examples of such binding phenomena are given in Webb \& Owen (2004b). We note that, In vivo, Delta-Notch binding and the resulting Delta production will be dependent on the cell's biochemical and biophysical environment. We consider microscale patterning in response to macroscale variation in tissue stimulation, providing motivation for the consideration of both spatial and temporal variation in the parameters associated with the feedback functions $f$ and $g$. Below, we investigate the emergence of fine-grained patterning in response to variation of these parameters.

\subsection{Patterning and stability - bifurcation analysis}

We first wish to determine under which parameter regimes fine-grained patterning will be produced by this model, with adjacent cells adopting different fates. Since the juxtacrine signalling mechanism under consideration is local, analysing the model equations for a twocell domain with periodic boundary conditions gives insight into the period-two patterning behaviour in more extensive arrays, and their analysis therefore forms an important precursor to what follows. The system (1) then reduces to the following four coupled equations, for which the solutions are either homogeneous or patterned with a wavelength of two cells:

$$
\begin{array}{ll}
\dot{d}_{1}=\lambda\left(g\left(n_{1}\right)-d_{1}\right), & \dot{d}_{2}=\lambda\left(g\left(n_{2}\right)-d_{2}\right), \\
\dot{n}_{1}=f\left(d_{2}\right)-n_{1}, & \dot{n}_{2}=f\left(d_{1}\right)-n_{2} .
\end{array}
$$

Clearly, from (4) and (5), the steady states are solutions of:

$$
\sigma=\phi(\phi(\sigma))
$$

where the notation $\phi(\sigma)=f(g(\sigma))$ has been introduced for concision. Via linear stability analysis, it is straightforward (see Collier et al. (1996)) to show that the homogeneous steady state is unstable and a pair of heterogeneous steady states exist if

$$
f^{\prime}\left(d^{*}\right) g^{\prime}\left(n^{*}\right)<-1
$$

where $\left(n^{*}, d^{*}\right)$ is the homogeneous steady state satisfying $n^{*}=\phi\left(n^{*}\right), d^{*}=g\left(n^{*}\right)$ and ' denotes differentiation.

The transition from a regime in which the homogeneous steady state is stable, corresponding to adjacent cells having identical fates, to that in which additional solutions to (6) are generated leads to the creation of fine-grained patterns (with adjacent cells reaching different fates via the appropriate solution branch). Figure 1(a) shows this transition in response to variations of the parameter $a$, which controls the Delta-Notch binding rate, indicating how patterns in Notch activation are created by local, symmetric divergence in 
each of the two cells at a pair of pitchfork bifurcations, enclosing a region of parameter space in which period two patterns are generated. The pitchfork behaviour reflects the symmetry of (4), (5) under the transformation $n_{1} \leftrightarrow n_{2}, d_{1} \leftrightarrow d_{2}$. The bifurcation points are denoted $a_{1}^{*}$ and $a_{2}^{*}$ (for $a_{1}^{*}<a_{2}^{*}$ ) and a corresponding bifurcation diagram is of course also obtained for the levels of Delta activation in each cell (not shown). Figure 1(b) shows the positions of the bifurcation points $a_{1}^{*}$ and $a_{2}^{*}$ in $(a, b)$ parameter space, illustrating how the pitchfork bifurcation structure illustrated in Figure 1(a) changes for illustrative values of $k=h$ (similar behaviour is observed for $k \neq h$, but is not shown). We observe that, in contrast to the enclosed patterning region $a \in\left(a_{1}^{*}, a_{2}^{*}\right)$ shown in Figure 1(a), variation of $b$ induces a single patterning bifurcation for fixed $a, k, h$. Qualitatively similar behaviour to that shown in Figure 1(b) is observed under simultaneous variation of $b$ and $k=h$ (for fixed $a$ ) indicating that a pair of pitchfork bifurcations enclosing a patterning region similar to that shown in Figure 1(a) is obtained under variation of $k, h$. Figure 1(b) also indicates that, as $b$ is decreased, the two bifurcations shown in Figure 1(a) approach each other and the pattern-forming region disappears; as the patterning region shrinks, the amplitude of Notch up- and down-regulation decreases until only the stable homogeneous steady state remains. Figure 2 shows how the number of solutions to (6) varies with $a$, and illustrates that near the bifurcation point we have $\sigma \approx \phi(\phi(\sigma))$ for a relatively wide range of $\sigma$ : this proves to be significant in terms of the range of applicability of the multiscale approach (see Appendix B).

The feedback parameter values employed in the numerical simulations presented in this work are based on those chosen by Collier et al. (1996) ( $b=100, a=0.01, k=h=2)$. Whilst no explicit discussion of the biological motivation for these choices was given there, Figure 1 indicates that these parameter choices provide a large disparity between the upand down-regulated states, consistent with the postulate that low levels of Notch activation lead to the adoption of the primary fate, high levels relegating the cell to the secondary fate. We highlight that in the following, we will employ the terms "up- or down-regulated" to refer to the level of Notch activation.

Figure 3 shows a typical numerical solution obtained for a line of cells in the parameter regime for which the criterion (7) applies. A striking feature of the Delta-Notch model is the robustness of the fine-grained (period-two) pattern. Collier et al. (1996) present simulations similar to Figure 3, remarking that defects in the alternating pattern tend to occur at irregular intervals along the cell string and take the form of adjacent cells displaying high Notch activity. Plahte (2001) analysed the alternating pattern and showed that in a line of cells at most two cells in succession can express high levels of Notch, breaking the periodic pattern.

We remark that longer-wavelength patterns are not prohibited in this one-dimensional system. For example, it is straightforward to show that the uniform state is unstable to period-three patterns if $f^{\prime}\left(d^{*}\right) g^{\prime}\left(n^{*}\right)<-2$ (see Collier et al. (1996)). Numerical simulations (omitted) indicate that these patterns are stable to periodic and aperiodic perturbations but that their domain of attraction is small and therefore are only observed for suitable initial data. (In the following we refer to this 'locally stable' behaviour.) Such patterns grow more slowly from the uniform state than those of period two; indeed, linear stability analysis reveals that the period-two pattern is the fastest-growing mode, providing an explanation for its robustness (Collier et al., 1996; Plahte, 2001). While the period-two pattern is distinguished by its dominance, the consideration of other patterning modes is nonetheless significant in related cell-signalling studies; for instance, in arrays of hexagonal cells, the fine-grained patterning regime corresponds to patterns containing three cells rather than 


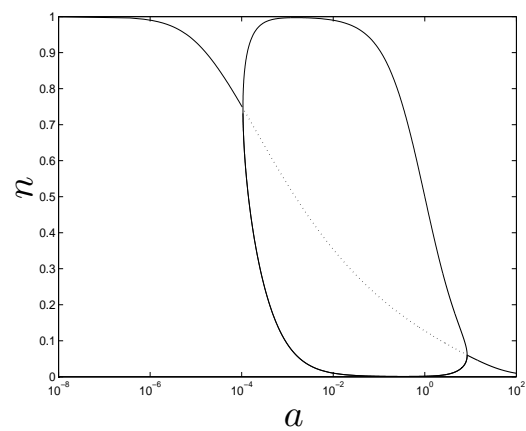

(a)

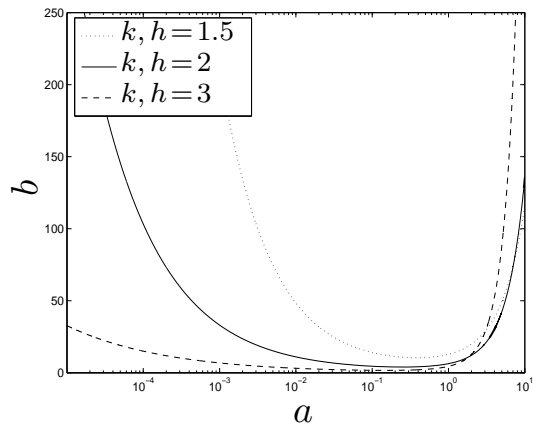

(b)

Figure 1: Bifurcation diagrams for the system (1) in a pair cells within a periodic array. In (a) the level of Notch activity in either of the two cells is represented by the appropriate solution branch: the two stable solution curves in the central region correspond to the period-two patterned state, one cell being in each of the two states shown. Unstable states are represented by dotted lines. For the particular parameters chosen $(\lambda=1, b=100, k=$ $h=2$ ), the bifurcation points shown in (a) are $a_{1}^{*} \approx 1.07 \times 10^{-4}$ and $a_{2}^{*} \approx 8.41$. In (b) the positions of bifurcation points $a_{1}^{*}, a_{2}^{*}$ under variation of $b$ are shown; the line-styles denote sample values of $k=h$.

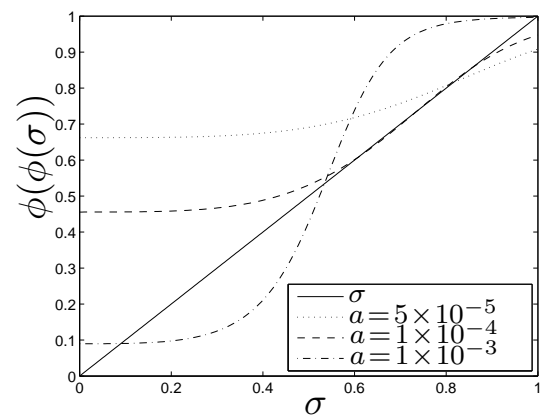

Figure 2: Illustrative plots of the function $\phi(\phi(\sigma))$ indicating the number of solutions to (6) in the regimes $a<a_{1}^{*}, a \approx a_{1}^{*}$ and $a>a_{1}^{*}$. Qualitatively similar plots are obtained near $a=a_{2}^{*}$. The remaining parameter values are as in Figure 1 .

two. In addition, microscopic patterns of considerably longer wavelength can be observed in developing tissues and Webb \& Owen (2004b) have shown that such patterns may be generated in related models of lateral inhibition. Multiscale analyses of such considerations will be presented elsewhere.

The bifurcation analysis of larger strings of cells is complicated by the increasing number of patterning branches that emerge as the string becomes longer. For example, in a string of three cells bifurcation analysis reveals that there are three possible pattern types: (i) two identically up-regulated cells and one down-regulated, (ii) two identically down-regulated cells and one up-regulated and (iii) one up-regulated cell, one down-regulated and one intermediate. Example bifurcation diagrams of period-three patterns in a string of three cells are shown in Figure 4, indicating that type (i) and (ii) patterns branch subcritically from the homogeneous steady state and that an additional branch of type (iii) patterns 


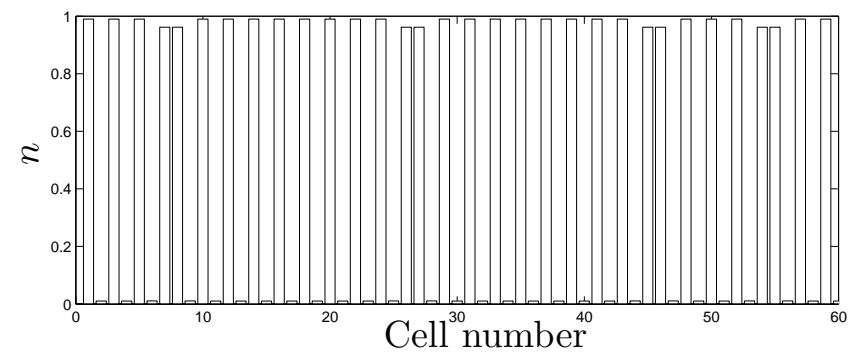

Figure 3: Typical numerical simulation (run to steady state) showing the level of Notch activity in a line of 60 cells. Initial conditions for Notch and Delta activity are small random deviations around the (unstable) homogeneous steady state. Parameter values as in Figure 1 with $a=0.01$.

connecting the various configurations of type (i) and (ii) is created as $k=h$ is increased. We emphasise that 'stability' as indicated in Figure 4 refers to stability within the three-cell model, which, in particular, precludes the existence of period-two solutions.

More generally, our investigations indicate that the system may be driven to patterning modes of period greater than two by appropriate choice of periodic initial conditions and domain size. In the case of periodic initial data of odd wavelength (which are not themselves a steady state), the system evolves to a pattern of this odd period. The resulting patterns are locally stable in the sense outlined above. Initial data of even period seems always to result in the emergence of the period-two pattern. This behaviour further exemplifies the robustness of the alternating pattern: longer range patterns of even period appear to form only from repetition of the stable period-two pattern, whilst locally-stable patterns of odd period are only observed for suitable initial data, the behaviour being dominated by the alternating (period-two) state. An investigation of the longer-range pattern-forming behaviour of this and related signalling models in more extensive cellular arrays will be presented elsewhere.

Aside from the linear-instability criterion discussed above, Plahte \& Øyehaug (2007) give two further possible mechanisms to explain the dominance of the period two pattern: (i) the feedback structure of the model (1) fundamentally favours this pattern, independent of the specific choices of the feedback functions $f$ and $g$ : the apparent negative feedback loop actually consists of a sequence of interlaced positive loops and negative loops (more detail is given in Plahte (2001)) that drive Delta and Notch activity into alternate extreme states, stabilised by the negative loops; (ii) the period-two pattern is the only pattern that can be produced by a travelling wave invading the unstable homogeneous state.

To summarise, the dominant pattern in the one-dimensional juxtacrine signalling model of Collier et al. (1996) is that of period two, consistent with the fine-grained patterning observed in the biological system this model aims to represent. Linear analysis, first presented by Collier et al. (1996), provides a simple condition that identifies the bifurcation of such patterns from the homogeneous steady state and reveals that this is the fastest-growing mode of the linearisation around the uniform state. Additional analysis by Plahte (2001) and Plahte \& Øyehaug (2007) provides intuition into the dominance of the fine-grained pattern. In this section, we have shown in detail how period-two pattern emergence is dictated by variation of the parameters associated with the feedback strength. The complications arising when considering longer-range patterns are discussed and the bifurcation structure associated with period-three patterns illustrated. In addition, we note that at- 


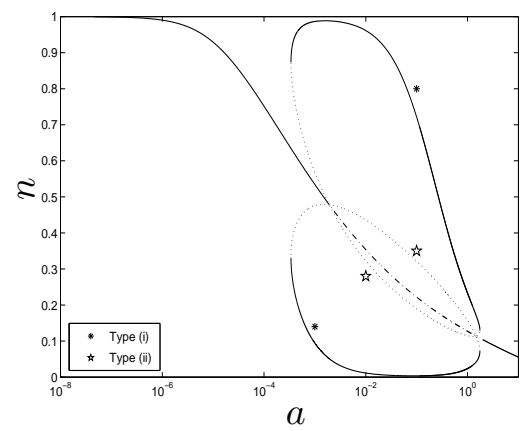

(a)

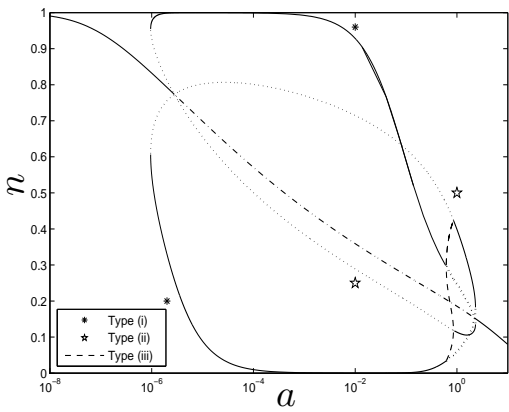

(b)

Figure 4: Bifurcation diagrams for the system (1) in a string of three cells with periodic boundary conditions. Unstable patterned states are indicated by dotted lines and the unstable homogeneous state by a dot-dashed line. Parameter values as in Figure 1 except in (b) $k=h=3$. In (a), only type (i) and (ii) patterns exist: in the central region, the upper branch indicates the steady-state values of two of the three cells and the lower branch the remaining cell. Type (ii) patterns are always unstable, type (i) patterns branch subcritically from the two bifurcation points. Pane (b) indicates similar patterning behaviour to that in (a) with the addition of a stable type (iii) branch (indicated by a dashed line) connecting small regions of stable type (ii) patterns.

tempts to generate longer-wavelength patterns of even period result simply in repetition of the alternating state and that patterns of odd period are observed only in simulations for appropriate initial data, further demonstrating the dominance of the fine-grained pattern.

Via a multiscale analysis, in the following sections, we construct a continuum model suitable to describe the generation of fine-grained patterns due to variations in feedback strength near either bifurcation point $a_{1}^{*}$ or $a_{2}^{*}$.

\section{A multiscale analysis of Delta and Notch activity in a line of cells}

\subsection{Model formulation}

In this section, we employ a multiscale method to derive systematically a continuum model based upon the discrete system (1) and capable of describing the dominant fine-grained (period-two) patterning phenomena examined in $\S 2.2$. Figure 1 suggests that introducing appropriate spatial variation of one (or more) of the model parameters can result in patterning being induced in certain regions of the domain only, and this generalisation from a uniform choice of parameters will be instructive in terms of the development of the methodology. In a biological context, such parameter variation would correspond to differences in the sensitivity of the cells to Delta-Notch binding, say, leading to the adoption of a particular programme of gene activation by a subset of the cell population according to spatial position. The results that we shall derive for spatially non-uniform binding sensitivity include the uniform case as a particular example, and our analysis will also include explicit treatment of the latter.

To construct such a continuum model, a homogenisation process is required. In order 


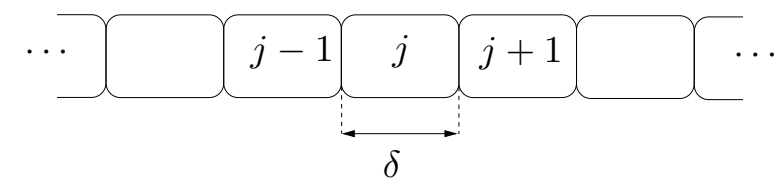

$\rightarrow x=\delta j$

Figure 5: Definition sketch: a line of cells and the spatial coordinates employed in the multiscale analysis.

that the local periodicity of the system be preserved, the variation of the parameter chosen to be spatially non-uniform is assumed to be slow compared to the variations of Delta and Notch activity; that is, we construct a two-scale model. The model is derived as follows. Considering a line of cells, we denote the size of cells by $\delta \ll 1$, introduce a slowly-varying continuum variable $x=\delta j$ and represent the levels of Delta and Notch activity in the multiple-scales form $d_{j}(t)=d(j, x, t), n_{j}(t)=n(j, x, t), j$ being the fast, and $x$ the slow, spatial variable (see Figure 5).

We remark here that a naïve and ad hoc continuum limit of the discrete model (1) obtained via the assumption of slow variation $\left(d_{j}=d(x, t), n_{j}=n(x, t)\right)$ takes the form

$$
\frac{\partial d}{\partial t}=\lambda(g(n)-d), \frac{\partial n}{\partial t}=f(d)-n+\frac{f^{\prime}(d)}{2} \frac{\partial^{2} d}{\partial x^{2}} .
$$

Such a model is incapable of capturing fine-grained patterning; in the following, we shall derive a substantially different continuum model which accommodates such short-range phenomena. Indeed, a linear-stability analysis of (8) readily shows it to be ill-posed, with the growth rate of a perturbation to the uniform steady state increasing without bound as its wavelength tends to zero, implying that the continuum limit is inapplicable. Further insight into this and to what follows can be obtained by considering the special case of (1) in which $f$ and $g$ are suitably redefined to be odd functions (and $n_{j}$ and $d_{j}$ are translated by their steady state values): setting:

$$
d_{j} \rightarrow(-1)^{j} d_{j}, n_{j} \rightarrow(-1)^{j+1} n_{j},
$$

we recover $(1)$ with $g\left(n_{j}\right)$ replaced by $-g\left(n_{j}\right)$ (so that $g$ is then to be viewed as an increasing function of $n_{j}$ ); with this change of sign, (8) becomes well-posed and, in addition, this special case provides useful guidance for the systematic calculations that follow.

The variation in feedback strength due to macroscale biochemical or biophysical conditions is for definiteness modelled by introducing a slow spatial variation to the parameter $a$ that controls the response of the cell to Delta activity in neighbouring cells (keeping the others fixed for simplicity) - i.e. we set $a=a(x)$. Since we intend to induce differences in patterning behaviour in the domain, it is natural to expand about one of the bifurcation points $a=a_{1}^{*}, a_{2}^{*}(c f$. Figure 1$)$ :

$$
a(x ; \delta)=a^{*}+\delta^{2} A(x),
$$

where $a^{*}$ denotes either bifurcation point. In the case $a^{*}=a_{2}^{*}$, for constant $A<0$, heterogeneous steady states exist and patterning results, whilst for $A \geqslant 0$, the homogeneous solution is stable. For $a^{*}=a_{1}^{*}$ the converse is true.

Since neighbouring cells are expected to differ significantly in their Delta and Notch activity in regimes in which patterning occurs (see Figures 1 and 3), we introduce the 
following notation to differentiate between "odd" and "even" cells:

$$
\begin{array}{lll}
d(j, x, t)=d^{+}(x, t), & n(j, x, t)=n^{+}(x, t): & j=2 m+1, \\
d(j, x, t)=d^{-}(x, t), & n(j, x, t)=n^{-}(x, t): & j=2 m,
\end{array}
$$

for integer $m$. On expanding in Taylor series, the spatial coupling term $\bar{d}_{j}$ may be written:

$$
\bar{d}_{j}=\frac{d_{j+1}+d_{j-1}}{2}=\bar{d}^{ \pm}(x, t)=d^{\mp}(x, t)+\frac{\delta^{2}}{2} \frac{\partial^{2}}{\partial x^{2}} d^{\mp}(x, t)+\mathcal{O}\left(\delta^{4}\right),
$$

according to whether $j$ is odd or even. Exploiting this notation, in the following we derive coupled equations governing Delta and Notch activity in odd and even cells separately, facilitating the inclusion of fine-grained patterning phenomena within our continuum model, thereby simplifying the multiscale analysis considerably.

Since the bifurcation is of pitchfork type (because in the patterned state either the even or the odd cells can be upregulated), we expect $\left(d^{ \pm}, n^{ \pm}\right)=\left(d^{*}, n^{*}\right) \pm \mathcal{O}\left(\sqrt{a^{*}-a}\right)$ as $a \rightarrow a^{*}$. We therefore consider an expansion of the form:

$$
\begin{aligned}
& d^{ \pm}(x, t ; \delta)=d^{*}+\delta d_{1}^{ \pm}(x, t)+\delta^{2} d_{2}^{ \pm}(x, t)+\cdots, \\
& n^{ \pm}(x, t ; \delta)=n^{*}+\delta n_{1}^{ \pm}(x, t)+\delta^{2} n_{2}^{ \pm}(x, t)+\cdots,
\end{aligned}
$$

where $d^{*}, n^{*}$ are the homogeneous steady states.

We wish to analyse the equations at the timescale on which spatial coupling enters the at $\mathcal{O}(\delta)$; rescaling time according to $\tau=\delta^{2} t$ achieves this ${ }^{1}$. Combining the above, from (1), we find that Delta activity is governed by

$$
\begin{aligned}
& \mathcal{O}(1): 0=g\left(n^{*}\right)-d^{*}, \\
& \mathcal{O}(\delta): 0=\mathscr{L}\left(n_{1}^{ \pm}, d_{1}^{ \pm}\right), \\
& \mathcal{O}\left(\delta^{2}\right): 0=\mathscr{L}\left(n_{2}^{ \pm}, d_{2}^{ \pm}\right)+\frac{n_{1}^{ \pm 2}}{2} g^{\prime \prime}\left(n^{*}\right), \\
& \mathcal{O}\left(\delta^{3}\right): \frac{1}{\lambda} \frac{\partial d_{1}^{ \pm}}{\partial \tau}=\mathscr{L}\left(n_{3}^{ \pm}, d_{3}^{ \pm}\right)+\frac{g^{\prime \prime \prime}\left(n^{*}\right) n_{1}^{ \pm 3}}{6}+g^{\prime \prime}\left(n^{*}\right) n_{1}^{ \pm} n_{2}^{ \pm},
\end{aligned}
$$

and Notch by

$$
\begin{aligned}
& \mathcal{O}(1): 0=f\left(d^{*}\right)-n^{*}, \\
& \mathcal{O}(\delta): 0=\mathscr{M}\left(n_{1}^{ \pm}, d_{1}^{\mp}\right), \\
& \mathcal{O}\left(\delta^{2}\right): 0=\mathscr{M}\left(n_{2}^{ \pm}, d_{2}^{\mp}\right)+F_{1}+\frac{d_{1}^{\mp 2}}{2} f^{\prime \prime}\left(d^{*}\right), \\
& \mathcal{O}\left(\delta^{3}\right): \frac{\partial n_{1}^{ \pm}}{\partial \tau}=\mathscr{M}\left(n_{3}^{ \pm}, d_{3}^{\mp}\right)+\frac{f^{\prime \prime \prime}\left(d^{*}\right) d_{1}^{\mp 3}}{6}+G_{1} d_{1}^{\mp}-\frac{f^{\prime}\left(d^{*}\right)}{2} \frac{\partial^{2} d_{1}^{\mp}}{\partial x^{2}}+f^{\prime \prime}\left(d^{*}\right) d_{1}^{\mp} d_{2}^{\mp} .
\end{aligned}
$$

Here $\mathscr{L}$ and $\mathscr{M}$ are linear operators defined by $\mathscr{L}(\eta, \xi)=\eta g^{\prime}\left(n^{*}\right)-\xi, \mathscr{M}(\eta, \xi)=-\eta+f^{\prime}\left(d^{*}\right) \xi$ and $F_{1}, G_{1}$ are the $\mathcal{O}\left(\delta^{2}\right)$ perturbations to $f\left(d^{*}\right), f^{\prime}\left(d^{*}\right)$ expanded around $a=a^{*}$, defined as follows:

$$
F_{1}=A(x) \frac{\partial f}{\partial a}, \quad G_{1}=A(x) \frac{\partial^{2} f}{\partial d \partial a},
$$

\footnotetext{
${ }^{1}$ Considering the speed of a period-two pattern invading the unstable steady-state provides an alternative motivation for these scalings; see Appendix A.
} 
and evaluated at $a=a^{*}, d=d^{*}$.

Equations (16) and (20) are the definitions of the homogeneous steady states $\left(d^{*}, n^{*}\right)$; (17) and (21) provide relationships between $n_{1}^{ \pm}$and $d_{1}^{ \pm}\left(n_{1}^{ \pm}=f^{\prime}\left(d^{*}\right) d_{1}^{\mp}, d_{1}^{ \pm}=g^{\prime}\left(n^{*}\right) n_{1}^{ \pm}\right)$ and, noting that the bifurcation at which the alternating pattern is created occurs at $a=$ $a^{*}, f^{\prime}\left(d^{*}\right) g^{\prime}\left(n^{*}\right)=-1$, additionally yield $\left(d_{1}^{+}, n_{1}^{+}\right)=-\left(d_{1}^{-}, n_{1}^{-}\right)$so that Delta and Notch activity in neighbouring cells diverges symmetrically. The systems at $\mathcal{O}(\delta)$ and higher are degenerate, reflecting the fact that we are analysing the behaviour close to the bifurcation point,

$$
g^{\prime}\left(n^{*}\right) \mathscr{M}\left(n_{i}^{ \pm}, d_{i}^{\mp}\right)-\mathscr{L}\left(n_{i}^{\mp}, d_{i}^{\mp}\right)+\mathscr{L}\left(n_{i}^{ \pm}, d_{i}^{ \pm}\right)-g^{\prime}\left(n^{*}\right) \mathscr{M}\left(n_{i}^{\mp}, d_{i}^{ \pm}\right)
$$

being identically zero.

The $\mathcal{O}\left(\delta^{3}\right)$ equations (19), (23) may be reduced to a reaction-diffusion equation for $n_{1}^{+}$. Equation (17) then provides $d_{1}^{ \pm}=g^{\prime}\left(n^{*}\right) n_{1}^{ \pm}$which, together with $\left(d_{1}^{+}, n_{1}^{+}\right)=-\left(d_{1}^{-}, n_{1}^{-}\right)$ allows for a complete solution. Equations governing $d_{1}^{ \pm}$are obtained via an identical method. Employing the combination (25) to remove the $\mathcal{O}\left(\delta^{3}\right)$ terms $n_{3}^{ \pm}, d_{3}^{ \pm}$between (19) and (23), equations (18) and (22) to eliminate the $\mathcal{O}\left(\delta^{2}\right)$ terms $n_{2}^{ \pm}, d_{2}^{ \pm}$in favour of $d_{1}^{+}, n_{1}^{+}$and (17) yields:

$$
\Lambda \frac{\partial n_{1}^{+}}{\partial \tau}=\frac{\partial^{2} n_{1}^{+}}{\partial x^{2}}+\alpha n_{1}^{+}-\beta n_{1}^{+3}
$$

wherein $\alpha(x)$ and the constants $\Lambda$ and $\beta$ are defined by

$$
\begin{aligned}
\alpha(x) & =\frac{d^{* 2} A(x)}{\left(a^{*}+d^{* 2}\right)^{2}}\left(\frac{4 g^{\prime}\left(n^{*}\right)}{d^{*}} \frac{a^{*}-d^{* 2}}{a^{*}+d^{* 2}}+\left[g^{\prime}\left(n^{*}\right)\right]^{2} f^{\prime \prime}\left(d^{*}\right)+g^{\prime \prime}\left(n^{*}\right) f^{\prime}\left(d^{*}\right)\right), \\
\Lambda & =\frac{2(\lambda+1)}{\lambda}, \\
\beta & =\frac{\left[g^{\prime}\left(n^{*}\right)\right]^{4}\left[f^{\prime \prime}\left(d^{*}\right)\right]^{2}+\left[g^{\prime \prime}\left(n^{*}\right) f^{\prime}\left(d^{*}\right)\right]^{2}}{2}+\frac{\left[g^{\prime}\left(n^{*}\right)\right]^{3} f^{\prime \prime \prime}\left(d^{*}\right)+g^{\prime \prime \prime}\left(n^{*}\right) f^{\prime}\left(d^{*}\right)}{3} .
\end{aligned}
$$

The corresponding Delta activity in odd cells is given by equation (17). We remark that this equation is invariant under the transformation $n_{1}^{+} \leftrightarrow-n_{1}^{+}$, reflecting the symmetric divergence of activity in adjacent cells near the bifurcation point.

Equation (26) shows that, on the macroscale, the juxtacrine signalling mechanism manifests itself as linear diffusion (with effective diffusivity $\lambda / 2(\lambda+1)$ ), in keeping with the use of reaction-diffusion models for such cell-signalling systems. Under a more restrictive assumption on $f$ and $g$, a continuum approach applies in a wider parameter regime; the resulting partial differential equation is more strongly nonlinear, however, this analysis being included in Appendix B.

For constant $\alpha, \beta$, the uniform steady states of $(26)$ are $n_{1}^{+}=(0, \pm \sqrt{\alpha / \beta})$. We emphasise that the non-trivial states are "uniform" in the sense that they are constant in odd cells; in even cells $n_{1}^{-}=(0, \mp \sqrt{\alpha / \beta})$. For the parameter choices under consideration $(b=100$, $k=h=2) \beta>0$ at each bifurcation point $a_{1}^{*}, a_{2}^{*}$; inside the patterning region $a \in\left(a_{1}^{*}, a_{2}^{*}\right)$, $\alpha>0$, otherwise $\alpha \leqslant 0$. Considering only the local value of the feedback strength $\alpha=\alpha(x)$, we therefore expect the generation of patterned states to reflect the pitchfork bifurcations at $a_{1}^{*}$ and $a_{2}^{*}: n \sim n^{*} \pm \delta \sqrt{\alpha(x) / \beta}, d \sim d^{*} \pm \delta g^{\prime}\left(n^{*}\right) \sqrt{\alpha(x) / \beta}$. For brevity, hereafter we will refer to this patterning behaviour as that influenced by "local feedback strength".

In the following sections we show how simulations of the discrete system (1), with small and slow variation in feedback strength $\left(a=a^{*}+\delta^{2} A(x)\right)$, compare with the solutions to the homogenised approximation (26) and with the bifurcation behaviour predicted by considering local feedback strength only. 


\section{Numerical results}

\subsection{Simulations}

We now present numerical solutions of (26) on the domain $0<x<L$ in which we choose $A(x)=2 x / L-1$ to illustrate how macroscale spatial variation in feedback strength dictates the emergence of fine-grained patterning in the domain. We compare these to corresponding numerical simulations of the full discrete system as well as those predicted by considering the local feedback strength, in which case a pitchfork bifurcation is located at $x=L / 2$ $(A=0)$. Since we expect macroscale variation over the domain, the periodicity conditions which we imposed at $x=0, L$ in the previous simulations (see Figure 3 ) are replaced by no-flux conditions. For clarity we highlight that the parameter choices employed below correspond to analysing the generation of patterns near the bifurcation point $a^{*}=a_{2}^{*}$; corresponding behaviour is observed near $a_{1}^{*}$.

Numerical solution of equation (26) was achieved by discretising on a uniform spatial grid and employing the initial value problem solver ode15s in MATLAB. The grid spacing in the discretisation of (26) is $\Delta x=10^{-4}$; we note that the grid spacing is not prohibited from exceeding the diameter of a single cell, since the cell-scale variation has been systematically averaged out.

In Figure 6, the steady-state levels of Delta and Notch activity predicted by (26) are compared to a corresponding simulation of the discrete system (1). In each case, fine-grained patterning is obtained in the left half of the domain; in the right half of the domain, the perturbations decay slowly to zero. Also shown is the pitchfork bifurcation at $x=L / 2$ $(A=0)$ obtained from considering only local feedback. Figure 6 shows good qualitative agreement between the discrete simulation and the homogenised model; however, in each case, a smooth transition between patterning and spatial homogeneity imparted by the spatial coupling terms in (1) and (26) is observed in place of a pitchfork bifurcation. Far from $a=a^{*}$, considering only local feedback provides a good approximation to the behaviour of (1) and (26).

\subsection{Travelling-wave behaviour}

In this model, spatial patterning can be generated by a wave front, behind which a regular pattern forms that determines cell fate. Examples of such behaviour in biological systems include the so-called morphogenetic furrow in the retina of Drosophila, behind which a regular pattern of photoreceptors surrounded by support and pigment cells is created (Owen, 2002; Plahte \& Øyehaug, 2007).

Extensive studies of pattern-forming travelling-wave propagation in discrete signalling systems have been presented by (for example) Owen (2002) and Plahte \& Øyehaug (2007) and we therefore omit a detailed study of the travelling-wave behaviour of the discrete model. We consider the travelling waves created in the regime for which the feedback strength is constant and the homogeneous steady state is unstable $\left(a<a^{*}\right)$; comparison between the wave speed predicted by our homogenised model and the discrete system gives a quantitative indication of the accuracy of the former in reproducing the underlying signalling system; such behaviour may be analysed analytically.

Travelling-wave solutions to nonlinear diffusion equations of the general form $u_{t}=u_{x x}+$ $u(1-u)(1+a u)$ have been given extensive treatment in the literature and so we do not repeat any details here. It suffices to note that two distinct types of travelling wave fronts exist: for $-1 \leqslant a \leqslant 2$, the wave front is 'pulled' and the propagation speed is determined 


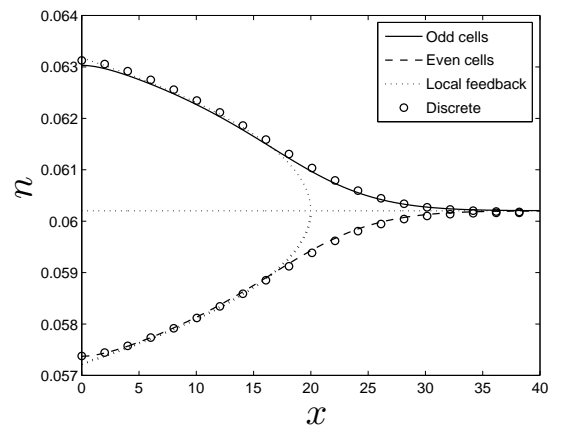

(a)

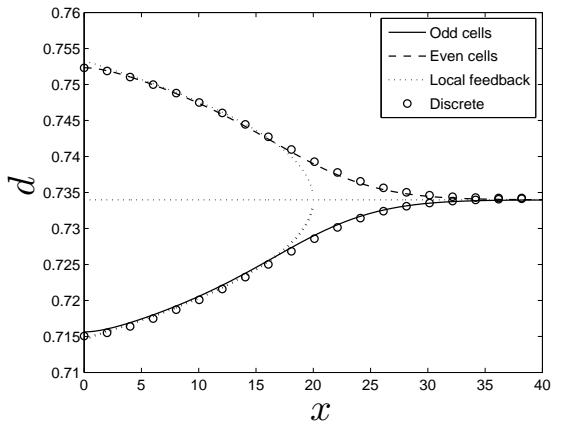

(b)

Figure 6: The steady-state levels of (a) Notch and (b) Delta activity on a domain of length $L=40, \delta=0.1$ (corresponding to 400 cells) predicted by (1), (26). The solid and dashed lines indicate the solution in odd and even cells, respectively, representing the long lengthscale envelope solutions arising from the multiple scales analysis. The dotted lines represent the homogeneous and heterogeneous steady states arising from local feedback only and the circles indicate the result from a corresponding simulation of the discrete model (1). Initial conditions are $n_{1}^{+}=0$ and small random fluctuations about $\left(n_{j}, d_{j}\right)=\left(n^{*}, d^{*}\right)$, respectively. Parameter values: $A(x)=2 x / L-1, b=100, k=h=2$ and the spatial mesh size in the discretisation is chosen as $\Delta x=1 \times 10^{-4}$.

by the leading edge; for $a>2$, the behaviour is termed 'pushed'. In the latter case, the propagation speed is determined by the whole wave front and converges uniformly to the linear propagation front $u\left(x+v t-x_{0}\right)$ for wavespeed $v$ and some $x_{0}$ (Hadeler \& Rothe, 1975; Stokes, 1976; Rothe, 1981). Equation (26) (under a suitable rescaling) corresponds to the choice $a=1$ and so, although we may obtain explicit travelling waves of the form

$$
n_{1}^{+}(x, t)=\frac{\sqrt{\alpha / \beta}}{1-\exp \left[ \pm \sqrt{\frac{\alpha}{2}}\left(x+x_{0} \mp 3 \sqrt{\frac{\alpha}{2 \Lambda^{2}}} t\right)\right]},
$$

the wave front in $(26)$ is pulled with speed $v<3 \sqrt{\alpha / 2 \Lambda^{2}}$. Instead, we calculate the wavespeed of 'linearly-selected' pattern-generating waves by analysing the behaviour at the leading edge via:

$$
n_{1}^{+}(x, t)=\delta N e^{k(x-v t)}
$$

where $N$ is an arbitrary constant. We obtain a dispersion relation, defining the wavespeed $v$ in terms of the wavenumber $k$. In conjunction with a repeated root condition, the minimum wavespeed is determined as $v=2 \sqrt{\alpha} / \Lambda$.

We induce a travelling wave of Delta and Notch activity by considering an initial state consisting of fine-grained patterning in part of the domain and an unstable homogeneous distribution in the remainder. As in $\S 4.1$, we impose no-flux conditions at $x=0, L$ in place of periodicity. We remark that the homogenised model admits true travelling waves, while those displayed by the original discrete system are modulated since the pattern generated behind the wave front is oscillatory. Thus, the analysis of pattern-generating waves is simplified by the continuum limit to a particularly large degree. Figure 7 depicts the modulated wave front in a simulation of (1), indicating how the period two pattern invades the homogeneous state, together with the travelling wave of Notch activity obtained from 
simulation of the homogenised model (26). Figure 8 shows the position of the midpoint of the wave front (defined as the position of the half-maximal value of Notch or Delta activity) as predicted by the homogenised and discrete models, together with the relative error of the former prediction, indicating that the numerical solution of the homogenised model faithfully reproduces the behaviour of the underlying discrete system; the minimum wavespeed $v=2 \sqrt{\alpha} / \Lambda$ of a linearly-selected wave provides a good approximation to this nonlinear behaviour. The deviation between the wavespeed $v$ and that obtained from simulation can be reduced by analysing travelling waves closer to the bifurcation point; in Appendix A the dependence of the travelling wave speed on $\delta$ in the discrete system (1) and the homogenised model are compared.

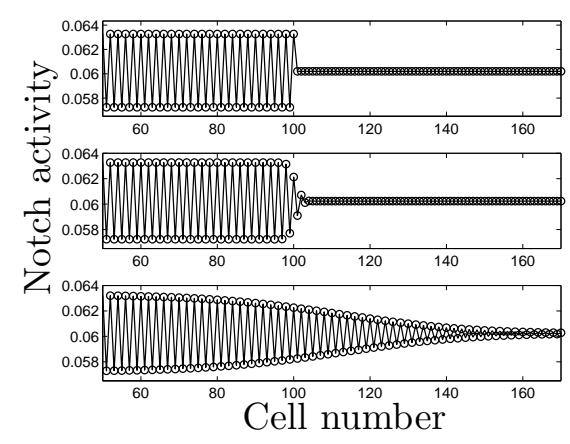

(a)

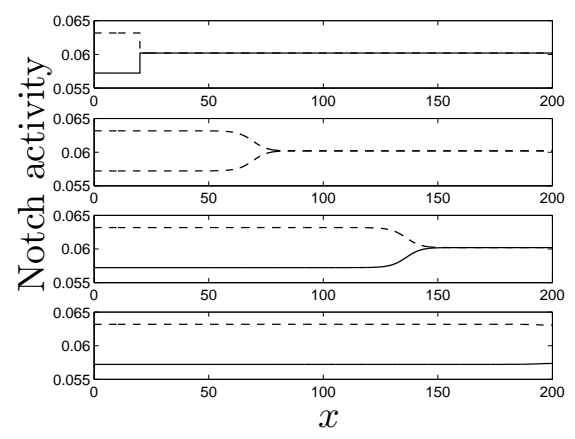

(b)

Figure 7: (a) The modulated travelling wave front in a simulation of (1) in a section of an array of 400 cells with initial conditions consisting of an "even" pattern (cells 1-100) and a homogeneous distribution in the remainder at $t=0,10,1000$; (b) travelling wave behaviour of the homogenised model $(26)$ at successive times $t=(0,3,6,10) \times 10^{4}$ on a domain of length $L=200, \delta=0.1$. The solid and dashed lines represent the levels of activity in odd and even cells, respectively. Parameter values as Figure 6 with $a=a^{*}-\delta^{2}$ (i.e. $A=-1$ ).

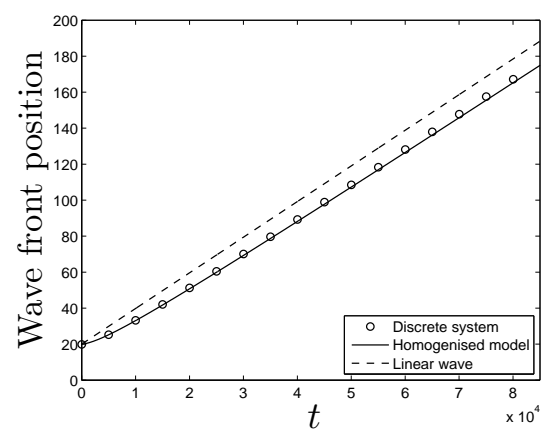

(a)

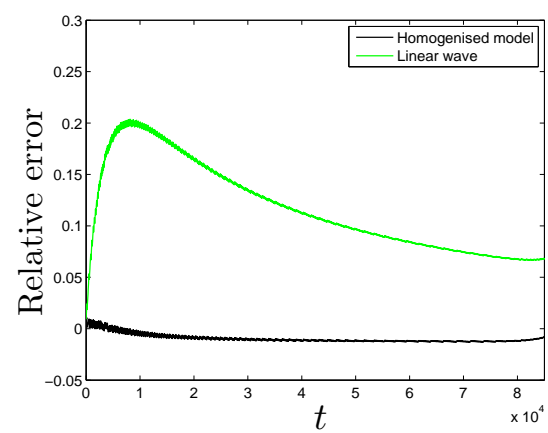

(b)

Figure 8: (a) The position of the midpoint of the travelling wave of Notch activity predicted by the homogenised and discrete models, and (b) the relative error between the wavefront position predicted by the homogenised and discrete model. Parameters as in Figure 7. 


\subsection{Competing patterns}

In the patterning regime, two heterogeneous steady states are stable: odd cells can exhibit high Notch activity whilst even cells have low activity (the "odd pattern"); conversely, even cells may exhibit high Notch activity (the "even pattern"). In the following, we demonstrate how the system (1) evolves from an initial state containing both types of pattern and we compare this against the behaviour of the homogenised model. In the latter, the odd pattern is represented by setting $n_{1}^{+}(x, 0)=\sqrt{\alpha / \beta}$ (other variables being calculated from $\left.d_{1}^{+}=g^{\prime}\left(n^{*}\right) n_{1}^{+},\left(n_{1}^{-}, d_{1}^{-}\right)=-\left(n_{1}^{+}, d_{1}^{+}\right)\right)$; conversely, the even pattern corresponds to $n_{1}^{+}(x, 0)=-\sqrt{\alpha / \beta}$.

Since the two patterns are equivalent up to a phase shift of a single cell, a priori, neither has a propensity to invade the other. Below, we consider in turn (i) the domain split evenly between odd and even patterns, and (ii) a region of even patterning enclosed by odd patterning to illustrate the differing behaviour. We remark that in the former case we impose no-flux boundary conditions at $x=0, L$; imposing periodicity renders the two scenarios equivalent.

Figure 9(a) shows that for initial conditions consisting of equal proportions of odd and even patterns, a smooth transition region is created, separating the two coexisting states. In the case of an asymmetric split, the initially-larger pattern region engulfs the smaller, as indicated by Figure 9(b) which shows the evolution from an initial state corresponding to a region of even patterning enclosed by odd. Here, the latter invades the former and the system eventually evolves to a state where only odd patterning exists. In each case, it is clear that the qualitative features of the discrete system are reproduced by the homogenised model.

We remark that sufficiently far from the bifurcation point, strong discrete effects prevent such engulfing behaviour and pinned patterning fronts are observed regardless of the proportion of the domain occupied by each pattern additional figure omitted for brevity). While Owen (2002) explored the propagation failure of waves in a similar model to that analysed here, smooth concentration profiles or invasion of an unstable state was considered; pinning between competing stable fine-grained pattern distributions has not been explored in detail in the literature. Our results indicate two distinct types of pattern competition dynamics within our simple signalling system; a more detailed examination of this issue in systems with more stable competing pattern modes will be given in a subsequent publication.

\section{Pattern formation in a two-dimensional array of cells}

In the preceding section, we have thoroughly analysed the fine-grained pattern forming behaviour of a string of cells, deriving a homogenised model which faithfully represents such behaviour. We now consider the behaviour of a two-dimensional array of square cells. Collier et al. (1996) showed that in two-dimensional arrays of hexagonal cells labelled $(i, j)$, patterns of period three in $i+j$ are possible; however, numerical simulations of the discrete system (1) in square cells indicate that checkerboard patterns (i.e., of period two in each coordinate direction) are robustly generated in arrays of square cells (data not shown). The analysis presented in the previous section may be exploited in this case so, for simplicity, we therefore concentrate on such patterns here. Investigation of discrete patterning behaviour in hexagonal cells, together with a multiscale analysis will be presented in a subsequent publication.

The macroscale model is constructed in an equivalent fashion to that outlined above: 


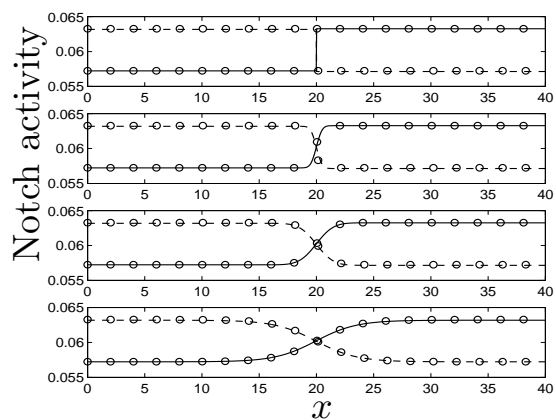

(a)

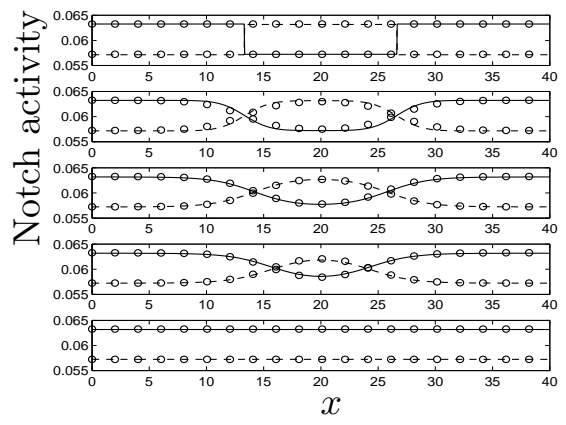

(b)

Figure 9: The behaviour of the systems (1), (26) at successive times in a line of 400 cells $(L=$ $40, \delta=0.1$ ) with initial conditions consisting of period two patterns with phase difference, $\pi$. In (a) the domain is split initially between the patterns equally: $t=0,30,250,5 \times 10^{3}$; in (b) odd patterns initially enclose a region of even patterning: $t=(0,0.05,0.45,0.95,1) \times 10^{5}$. The solid and dashed lines represent the levels of activity in odd and even cells predicted by the homogenised model (26) whilst the discrete simulation results are indicated by circles. Parameters as in Figure 7.

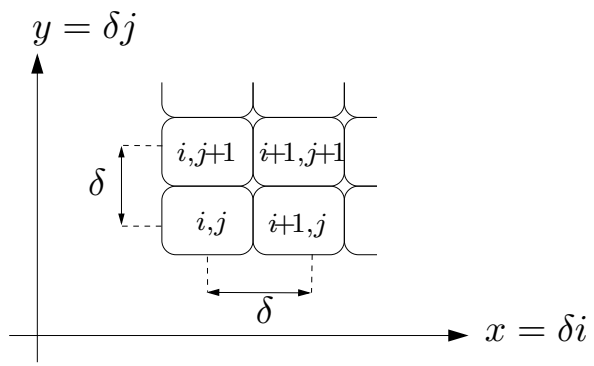

Figure 10: Definition sketch: an array of square cells and the spatial coordinates employed in the multiscale analysis. Note that in the discrete model (1) the aspect ratio of the cells is not explicitly captured so that square and rectangular cells are equivalent.

we consider an array of square cells, denote the horizontal and vertical distances between the cells by $\delta \ll 1$ (see Figure 10), introduce slowly-varying continuum variables $\boldsymbol{x}=$ $(x, y)=\delta(i, j)$ and represent the level of Delta and Notch activity in the multiple-scales form: $d_{i, j}(t)=d(i, j, \boldsymbol{x}, t), n_{i, j}(t)=n(i, j, \boldsymbol{x}, t)$. As in the one-dimensional case, we expand around either of the bifurcation points $a^{*}=a_{1}^{*}, a_{2}^{*}$ via

$$
a(\boldsymbol{x} ; \delta)=a^{*}+\delta^{2} A(\boldsymbol{x}),
$$

and, since neighbouring cells are expected to differ significantly in their Delta and Notch activity in regimes in which patterning occurs, we introduce the following notation:

$$
\begin{array}{lll}
d(i, j, \boldsymbol{x}, t)=d^{+}(\boldsymbol{x}, t), & n(i, j, \boldsymbol{x}, t)=n^{+}(\boldsymbol{x}, t) & i=2 m, j=2 m \\
d(i, j, \boldsymbol{x}, t)=\widetilde{d}^{+}(\boldsymbol{x}, t), & n(i, j, \boldsymbol{x}, t)=\widetilde{n}^{+}(\boldsymbol{x}, t) & i=2 m+1, j=2 m+1, \\
d(i, j, \boldsymbol{x}, t)=d^{-}(\boldsymbol{x}, t), & n(i, j, \boldsymbol{x}, t)=n^{-}(\boldsymbol{x}, t) & i=2 m+1, j=2 m \\
d(i, j, \boldsymbol{x}, t)=\widetilde{d}^{-}(\boldsymbol{x}, t), & n(i, j, \boldsymbol{x}, t)=\widetilde{n}^{-}(\boldsymbol{x}, t) & i=2 m, j=2 m+1
\end{array}
$$


for integer $m$. The spatial coupling term $\bar{d}_{i, j}$ may then be written:

$$
\begin{gathered}
\bar{d}_{i, j}=\frac{d_{i, j+1}+d_{i, j-1}+d_{i+1, j}+d_{i-1, j}}{4}=\frac{d^{\mp}(\boldsymbol{x}, t)+\widetilde{d}^{\mp}(\boldsymbol{x}, t)}{2} \\
+\frac{\delta^{2}}{4}\left\{\frac{\partial^{2}}{\partial x^{2}} d^{\mp}(\boldsymbol{x}, t)+\frac{\partial^{2}}{\partial y^{2}} \widetilde{d}^{\mp}(\boldsymbol{x}, t)\right\}+\mathcal{O}\left(\delta^{4}\right) .
\end{gathered}
$$

Lastly we consider an expansion of the dependent variables of the form:

$$
\begin{aligned}
& n^{ \pm}(\boldsymbol{x}, t ; \delta)=d^{*}+\delta n_{1}^{ \pm}(\boldsymbol{x}, t)+\delta^{2} n_{2}^{ \pm}(\boldsymbol{x}, t)+\cdots \\
& \widetilde{n}^{ \pm}(\boldsymbol{x}, t ; \delta)=d^{*}+\delta \widetilde{n}_{1}^{ \pm}(\boldsymbol{x}, t)+\delta^{2} \widetilde{n}_{2}^{ \pm}(\boldsymbol{x}, t)+\cdots
\end{aligned}
$$

with corresponding expansions for the Delta activity $d^{ \pm}$and $\widetilde{d}^{ \pm}$.

Under the additional rescaling $\tau=\delta^{2} t$, we obtain equations governing the Delta and Notch activity in each of the four cells of the same form as (16)-(23). We find $\left(d^{ \pm}, n^{ \pm}\right)=$ $\left(\widetilde{d}^{ \pm}, \widetilde{n}^{ \pm}\right),\left(d_{1}^{+}, n_{1}^{+}\right)=-\left(d_{1}^{-}, n_{1}^{-}\right)$and $d_{1}^{ \pm}=g^{\prime}\left(n^{*}\right) n_{1}^{ \pm}$(for brevity, we omit the details), confirming that due to the form of the nearest-neighbour interaction, only checkerboard patterns may exist in the patterning regime. A complete solution may therefore be obtained by deriving an equation for (e.g.) $n_{1}^{+}$. Multiscale analysis of striped patterns in a more complex model of ligand-receptor interaction (Owen \& Sherratt, 1998) will be presented elsewhere.

In the one-dimensional case, the model was expressed in terms of Delta and Notch activity in odd and even cells; here, we consider activity on "diagonal" cells $(i=j)$ and "off-diagonal" cells $(i+1, j ; i, j+1)$ and obtain a reaction-diffusion equation for $n_{1}^{+}$, the $\mathcal{O}(\delta)$ correction to Notch activity on diagonal cells. The remaining variables are given by $\left(n_{1}^{+}, g^{\prime}\left(n^{*}\right) n_{1}^{+}\right)=-\left(n_{1}^{-}, d_{1}^{-}\right)$. Following exactly the same method as $\S 3$, we obtain (unsurprisingly):

$$
\Lambda \frac{\partial n_{1}^{+}}{\partial \tau}=\frac{1}{2}\left(\frac{\partial^{2} n_{1}^{+}}{\partial x^{2}}+\frac{\partial^{2} n_{1}^{+}}{\partial y^{2}}\right)+\alpha n_{1}^{+}-\beta n_{1}^{+3},
$$

with $\Lambda, \alpha$ and $\beta$ defined by (28)-(29). The discrepancy between the effective diffusion coefficients in (26) and (40) is a consequence of the coupling term (2) which is scaled according to the number of neighbours surrounding a cell.

The behaviour of this model reproduces that analysed in detail in the one-dimensional case. For brevity, we therefore present only illustrative numerical simulations demonstrating the pattern-forming capability of this two-dimensional system and the correspondence between the discrete and homogenised models. So that patterning effects are visible, in Figure 11(a) the steady-state pattern in a small array of $30 \times 30$ cells $(\delta=0.5, L=15)$ is shown, illustrating the emergence of the checkerboard pattern under the feedback variation:

$$
A(\boldsymbol{x})=\frac{x+y}{L}-1 .
$$

As in the one-dimensional case, fine-grained patterning is obtained where $A<0$ and the solution tends smoothly to the homogeneous steady state as $a$ crosses the bifurcation point $a=a_{2}^{*}$ (corresponding behaviour is found near $a_{1}^{*}$ ). Figure 11(b) indicates that the homogenised model agrees well with the underlying discrete system, whilst the accuracy of the prediction based only the local feedback strength is poor.

In this two-dimensional system, an identical analysis to that presented in $\S 4.2$ reveals linearly selected diagonally-travelling pattern-generating waves travel with wavespeed $v=$ 


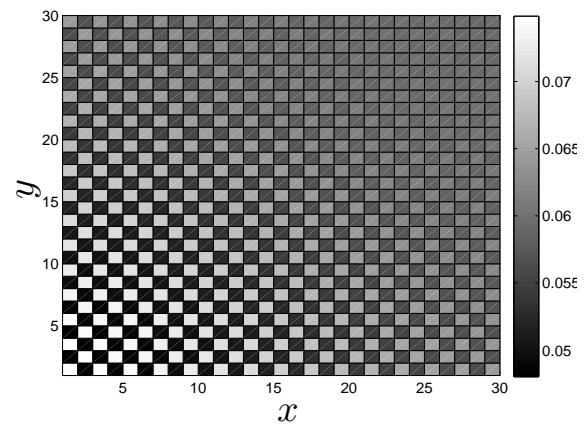

(a)

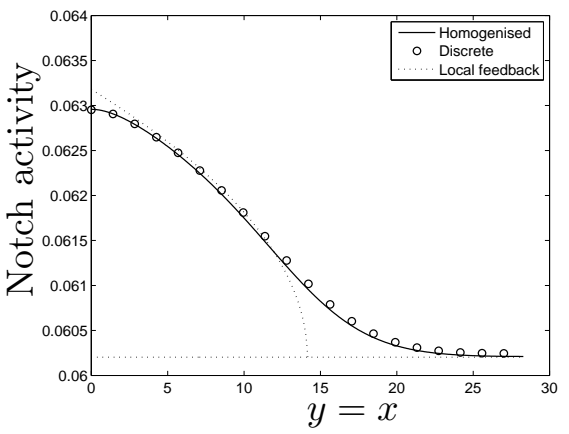

(b)

Figure 11: (a) The steady state level of Notch activity predicted by the discrete model (1) in a two-dimensional array of $30 \times 30$ cells $(\delta=0.5)$, and (b) a comparison between the levels of Notch activity on diagonal cells indicated by the discrete and homogenised models in an array of $300 \times 300$ cells $(\delta=0.1)$ along the line $y=x$. The dotted line indicates the steady state arising from local feedback only. $A(\boldsymbol{x})$ defined by (41), other parameters as in Figure 6 except $\delta$ as stated. (40) is solved using a spatial discretisation $\Delta x=1 \times 10^{-4}=\Delta y$.

$\sqrt{2 \alpha} / \Lambda$; Figure 12 shows a comparison between the midpoint of the wavefront (travelling along $y=x$ ) predicted by the numerical simulations of the discrete and homogenised model as well as that predicted by the linear analysis. Clearly, the homogenised model provides an excellent approximation to the behaviour of the discrete system; the linear prediction performs less well. Reducing $\delta$ (see appendix A) improves the accuracy of the linear approximation.

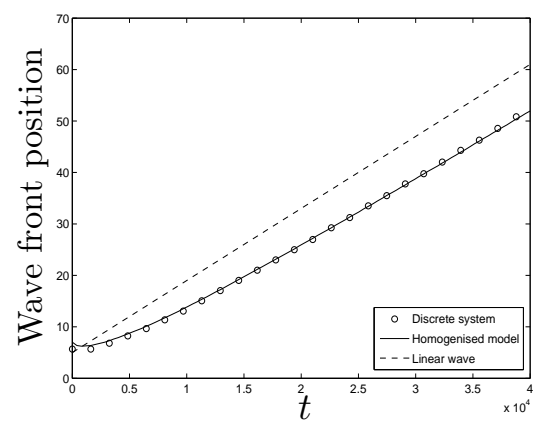

Figure 12: The position of the midpoint of the wavefront (travelling along $y=x$ ) of Notch activity predicted by the two-dimensional homogenised and discrete models on a square domain of side $L=100, \delta=0.1$ (corresponding to $1000 \times 1000$ cells). Initial conditions comprise a region of diagonal patterning $(0 \leqslant x \leqslant 5,0 \leqslant y \leqslant 5)$ surrounded by the homogeneous state. Parameters as in Figure 11.

Competing patterns also display behaviour corresponding to that observed in the onedimensional case. Figure 13 shows that a region of "diagonal patterning" (in which diagonal cells exhibit high levels of Notch) surrounded by an "off-diagonal" pattern is engulfed, evolving to a state with only the initially larger pattern remaining. Also shown is the position of the engulfing wave front in the discrete simulation (the position of the point 
of equal Notch activity in odd and even cells - i.e., $\left.n(\boldsymbol{x}, t)=n^{*}\right)$, indicating that the enclosed region becomes circular as is the case in the continuum limit (we note that the region of diagonal patterning becomes square again as it approaches extinction, reflecting the breakdown of the continuum limit). Due to invasion from all four directions and the smaller domain employed, the diagonal pattern region is replaced with off-diagonal more quickly than in the corresponding one-dimensional case. Competing patterns with only one grain boundary exhibit behaviour corresponding to that shown in Figure 9 (results omitted).

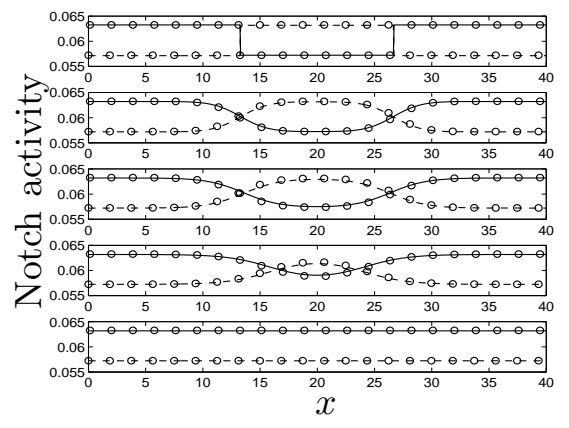

(a)

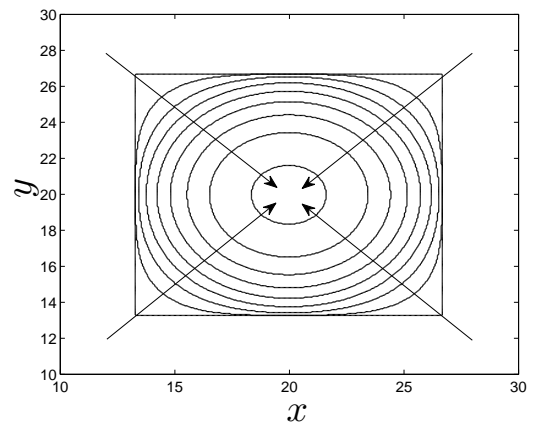

(b)

Figure 13: (a) The behaviour of the systems (1), (40) plotted along the line $y=15$ at successive times $t=(0,0.45,0.75,1.7,2.5) \times 10^{4}$ in a square domain of side $L=30$ $(\delta=0.1)$ cells with initial conditions of consisting of an off-diagonal pattern of Notch and Delta activity enclosing a square region of diagonal patterning. The solid and dashed lines represent the levels of activity in diagonal and off-diagonal cells predicted by the homogenised model (26) whilst the discrete simulation results are indicated by circles; (b) contours showing the position of the point of equal Notch activity in odd and even cells at $t=(0,0.4,0.6,0.8,1,1.2,1.4,1.6) \times 10^{4}$. Parameters as in Figure 7.

\section{Discussion}

In this paper, we have employed a multiscale technique to construct a continuum model which is capable of describing the fine-grained patterning which arises in certain parameter regimes associated with signalling feedback strength within the Delta-Notch signalling model of Collier et al. (1996). While many authors have considered the derivation of continuous models from an underlying discrete systems (indeed, Roussel \& Roussel (2004) consider a similar signalling model to that analysed here obtaining a reaction-diffusion equation provided that the variation in signalling molecule concentration is smooth), here we formulate a continuum model capable of describing variation in signalling activity between adjacent cells. In the main, such systems have hitherto been analysed using discrete techniques, whilst continuum models have been restricted to smooth concentration profiles or travelling wave behaviour of juxtacrine activation. Via the assumption of slow variation in feedback strength and judicious choice of variables, we derive a semilinear partial differential equation governing receptor and ligand activity on alternate cells under particular assumptions on the model parameters. The governing equations obtained demonstrate that on the macroscale, a juxtacrine signalling mechanism in a line of cells does in fact manifest itself as linear diffusion, even in the regime of fine-grained patterning. This provides motivation for the 
use of reaction-diffusion-type modelling of intercellular signalling. Under a more restrictive condition on the feedback functions specified in Collier et al. (1996), we derive a continuum model which applies under a wider parameter regime; this model is more strongly nonlinear however.

We have employed our model to investigate the emergence of patterns in a line of cells in response to slow spatial variation of the feedback strength within the Delta-Notch feedback mechanism and compared the model results to that predicted by corresponding simulations of the discrete system. Additionally we compare these numerical solutions to the bifurcation behaviour indicated by considering only the local vale of the feedback strength. It is evident that, near the bifurcation point, the homogenised model provides a much closer approximation to the behaviour of the underlying discrete signalling system than that predicted by analysis based upon the local feedback. Far from the bifurcation point, the local analysis provides a reasonable approximation to the behaviour. By analysing the evolution of travelling waves of Notch activity, we demonstrate excellent qualitative agreement between numerical solution of our homogenised model and the behaviour of the underlying discrete system. We also derive analytically the minimum wavespeed of a linearly selected travelling wave, indicating the (surprisingly wide) range of parameter values for which our continuum approximation applies. The evolution of two competing stable patterns was also investigated. Such behaviour is mathematically interesting and has not been examined thoroughly in the literature. Here, we indicate two distinct types of dynamics, as a precursor to a more comprehensive study. In particular, we show that, in general, near the bifurcation point, the system is unable to support coexistence of such patterns, evolving to contain only the pattern initially occupying the larger proportion of the domain; far from the bifurcation point, strong discrete effects allow pinning of such patterns. In the specific case of the domain being split equally between the pattern types, pinned behaviour is observed in both the continuum and discrete models; in general, such behaviour is precluded from the continuum representation. Further discussion is given in Appendix B.

By considering an array of square cells, we extended this formulation to derive a twodimensional continuum model which, since it supports checkerboard patterns only, is essentially equivalent to the one-dimensional model (the scaling of the coupling term provides a discrepancy) and corresponding behaviour was observed. Numerical simulations of finegrained pattern generation, travelling wave invasion and pattern competition were presented showing that the homogenised model faithfully reproduces the underlying discrete system.

To summarise, in the case of strong discrete effects, especially in the case of small cell populations, it is appropriate to treat cells as independent, obtaining a small system of ODEs to analyse; here, the converse limit has been identified and the appropriate mathematical description formulated and shown to be equivalent to PDE models of similar phenomena that are already well-understood. We remark that a disparity between the continuum and discrete representations of this system is revealed by considering a string of cells with periodic boundary conditions. Since the multiscale technique presented herein relies on a periodic unit of two (or, in two dimensions, four) cells, its application to an odd number of cells introduces a boundary layer of width on the order of a single cell at the domain edges; in contrast, the discrete model supports an arbitrary number of cells.

We have exploited the simple nature of this intercellular signalling model to derive a continuum model capable of describing fine-grained patterning which, nevertheless, does not infringe the requirement that the lengthscale of significant variation must be large in comparison to the cell length. The specific signalling model under consideration was cho- 
sen in large part for illustrative purposes; however, the mathematical techniques employed are applicable to more elaborate scenarios in other spatially discrete models. Considerations to be investigated elsewhere include more complicated signalling models, mechanisms which generate patterns with a longer characteristic scale (such as lateral induction, a corresponding juxtacrine mechanism with positive, rather than negative, feedback), multi-mode pattern competition and pinning, and the analysis of patterning behaviour in hexagonal cells.

\section{Acknowledgements}

This research was undertaken at The University of Nottingham; the authors gratefully acknowledge funding from BBSRC and EPSRC for this work (BB/D008522/1). JRK also acknowledges the support of the Royal Society and Wolfson Foundation. All bifurcation diagrams were generated in XPPAUT v5.91.

\section{A Travelling wave invasion}

In this appendix, we consider the invasion of the unstable homogeneous steady state $\left(n^{*}, d^{*}\right)$ by a linearly-selected modulated (period-two) travelling wave solution to (1). The invasion speed of such a wave is compared to a similar analysis on the continuum model (26) (see $\S 4.2$ ) and to simulations of the discrete system (1) to indicate the parameter range for which our continuum analysis is applicable. We remark that similar investigations have been presented in Plahte \& Øyehaug (2007), in which the dependence of the wavespeed on the feedback parameter $k=h$ (which controls the steepness of the sigmoid feedback functions) was considered and the results compared to corresponding wavespeeds obtained from simulations of a discrete system similar to (1).

We seek travelling wave solutions of the form

$$
\left(n_{j}, d_{j}\right)=\left(n^{*}, d^{*}\right)+\delta\left(n_{1}, d_{1}\right) e^{i \sigma t-k(j-c t)} ; \sigma, c \in \mathbb{R}, k \in \mathbb{C},
$$

where $\sigma$ is the linear growth rate, $k=k_{R}+i k_{I}$ is the complex wavenumber and $c$ is the wavespeed. From equations (1) we then obtain:

$$
\begin{aligned}
& k^{2} c^{2}+(2 i \sigma+(\lambda+1)) k c+\lambda\left(1-f^{\prime}\left(d^{*}\right) g^{\prime}\left(n^{*}\right) \cosh (k)\right)-\sigma(\sigma+i(\lambda+1))=0 \\
& 2 k c^{2}+c(2 i \sigma+\lambda+1)-\lambda f^{\prime}\left(d^{*}\right) g^{\prime}\left(n^{*}\right) \sinh (k)=0
\end{aligned}
$$

the second of which being a repeated root condition on the former dispersion relation. Numerical solution of these equations provides the speed of invading travelling wave together with the associated complex wavenumber (we note that we obtain $k=k_{R}+i \pi, \sigma=\pi c$, reflecting the period-two nature of the modulated invading wave). Comparison between the solution of (43) and (44), the corresponding linearly-selected wavespeed $v=2 \sqrt{\alpha} / \Lambda$ predicted by the homogenised model ( $\S 4.2)$, and numerical simulations of the discrete system (1) gives an indication of the range of applicability of the asymptotic analysis. We remark that the results presented in this appendix apply to the travelling waves near the bifurcation point $a_{2}^{*}$; corresponding results may be obtained at $a=a_{1}^{*}$.

We induce a travelling wave in the model (1) in the same way as described in $\S 4.2$, employing a string of 3000 cells and calculating the wavespeed from the middle part of the string to minimise boundary effects. Figure 14(a) shows how the predicted wavespeeds 
differ as $\delta$ is increased, indicating that both the linearly selected wave speed predicted by the continuum model (26) and that obtained from solution of (43) and (44) provide an excellent approximation to that observed in numerical simulations of the discrete system, even for parameter values far from the bifurcation point.

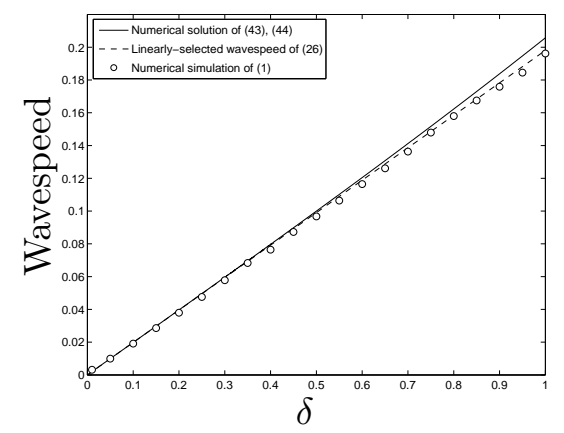

Figure 14: A comparison of the minimum linearly-selected wavespeed in the discrete system and continuum approximation (obtained via equations (43), (44) and, from (26), $v=2 \sqrt{\alpha} / \Lambda)$ and the observed wavespeed in simulations of the discrete system (1) at different values of $\delta\left(a=a_{2}^{*}-\delta^{2}\right)$. The remaining parameters are as in Figure 7 .

The solution of (43) and (44) may be simplified by considering the behaviour near the bifurcation points $a_{1}^{*}, a_{2}^{*}$ (denoted $a^{*}$ hereafter). In view of the expansion $a=a^{*} \pm \delta^{2}$ and the period two nature of the invading wave, we have $f^{\prime}\left(d^{*}\right) g^{\prime}\left(n^{*}\right)=-1+\mathcal{O}\left(\delta^{2}\right)$ and $k=\bar{k}+i \pi$ (where $0<\delta^{2} \ll \bar{k} \ll 1$ ); linearising, we obtain:

$$
\bar{k} \sim \sqrt{2\left(f^{\prime}\left(d^{*}\right) g^{\prime}\left(n^{*}\right)+1\right)}, \quad c \sim \frac{\lambda}{1+\lambda} \sqrt{2\left(f^{\prime}\left(d^{*}\right) g^{\prime}\left(n^{*}\right)+1\right)},
$$

motivating the scalings $x=\delta j, \tau=\delta^{2} t$ employed in $\S 3$.

\section{B Quasilinear continuum model for fine-grained pattern for- mation}

In this appendix, we derive a continuum model capable describing fine-grained patterns in a more general context via an approach in which odd and even cells are treated separately from the outset.

We consider a line of cells as illustrated by Figure 5, take the continuum limit, adopt the notation given by equations (11) and (12) for the levels of Delta and Notch activity in odd and even cells and scale time according to $\tau=\delta^{2} t$.

Employing the continuum version of equation (1), the spatial coupling term, $\bar{d}_{j}^{ \pm}$, may be written:

$$
\bar{d}_{j}^{ \pm}=g\left(n^{\mp}\right)-\delta \frac{\partial}{\partial x} g\left(n^{\mp}\right)+\delta^{2}\left(\frac{\partial^{2}}{\partial x^{2}} g\left(n^{\mp}\right)-\frac{1}{\lambda} \frac{\partial d^{\mp}}{\partial t}\right)+\mathcal{O}\left(\delta^{3}\right)
$$

A crucial assumption in the analysis that follows is that (to leading order) $\phi(\sigma)=f(g(\sigma))$ is its own inverse. As Figure 2 shows, this is the case near the bifurcation point $a=a^{*}$ at which the alternating pattern is created (see Figure 1). By choosing the nonlinearities $f$ and 
$g$ such that this condition is satisfied for all relevant $\sigma$ we are able to extend significantly the range of applicability of the continuum limit; specifically, we set:

$$
f(g(\sigma))=\phi(\sigma)+\delta^{2} \psi(\sigma) \text { with } \phi(\phi(\sigma))=\sigma,
$$

and introduce the expansions:

$$
\begin{aligned}
& d^{ \pm}(x, t ; \delta)=d_{0}^{ \pm}(x, t)+\delta d_{1}^{ \pm}(x, t)+\delta^{2} d_{2}^{ \pm}(x, t)+\cdots \\
& n^{ \pm}(x, t ; \delta)=n_{0}^{ \pm}(x, t)+\delta n_{1}^{ \pm}(x, t)+\delta^{2} n_{2}^{ \pm}(x, t)+\cdots
\end{aligned}
$$

The equations governing Notch activity on odd and even cells are then

$$
\begin{aligned}
\mathcal{O}(1): & 0=\phi\left(n_{0}^{ \pm}\right)-n_{0}^{\mp}, \\
\mathcal{O}(\delta): & 0=\phi^{\prime}\left(n_{0}^{ \pm}\right) n_{1}^{ \pm}-\frac{\partial}{\partial x} \phi\left(n_{0}^{ \pm}\right)-n_{1}^{\mp}, \\
\mathcal{O}\left(\delta^{2}\right): & \frac{\partial n_{0}^{ \pm}}{\partial \tau}=\psi\left(n_{0}^{\mp}\right)+\frac{n_{1}^{\mp 2}}{2} \phi^{\prime \prime}\left(n_{0}^{\mp}\right)+\frac{1}{2} f^{\prime \prime}\left(g\left(n_{0}^{\mp}\right)\right)\left(\frac{\partial}{\partial x} g\left(n_{0}^{\mp}\right)\right)^{2} \\
& +\left(\frac{\partial^{2}}{\partial x^{2}} g\left(n_{0}^{\mp}\right)-\frac{1}{\lambda} \frac{\partial d_{0}^{\mp}}{\partial \tau}\right) f^{\prime}\left(g\left(n_{0}^{\mp}\right)\right)-\frac{\partial}{\partial x}\left(n_{1}^{\mp} \phi^{\prime}\left(n_{0}^{\mp}\right)\right)+\phi^{\prime}\left(n_{0}^{\mp}\right) n_{2}^{\mp}-n_{2}^{ \pm} .
\end{aligned}
$$

These equations are written in terms of Notch activity only via $d_{0}^{ \pm}=g\left(n_{0}^{ \pm}\right)$. Employing (51) to eliminate $n_{1}^{ \pm}$and combining (52) to eliminate $n_{2}^{ \pm}$(noting that $\phi^{\prime}\left(n_{0}^{ \pm}\right) \phi^{\prime}\left(n_{0}^{\mp}\right)=1$ ), we obtain the following quasilinear diffusion equation for the leading-order Notch activity in even cells (dropping superscripts for brevity):

$$
\begin{aligned}
\lambda & \frac{\partial n_{0}}{\partial \tau}=\psi\left(\phi\left(n_{0}\right)\right)+\phi^{\prime}\left(\phi\left(n_{0}\right)\right) \psi\left(n_{0}\right)+\frac{\partial^{2} n_{0}}{\partial x^{2}} \\
& \left.+\left(\frac{\partial n_{0}}{\partial x}\right)^{2}\left\{\phi^{\prime}\left(\phi\left(n_{0}\right)\right) g^{\prime \prime}\left(n_{0}\right) f^{\prime}\left(g\left(n_{0}\right)\right)-\left[\phi^{\prime}\left(n_{0}\right) g^{\prime}\left(\phi\left(n_{0}\right)\right)\right]^{2} f^{\prime \prime}\left(g\left(\phi\left(n_{0}\right)\right)\right)\right)\right\} .
\end{aligned}
$$

We remark that a nonlinear diffusion term may be introduced by writing this in the standard form:

$$
\frac{\partial}{\partial t} \Phi\left(n_{0}\right)=\frac{\partial}{\partial x}\left(\Psi\left(n_{0}\right) \frac{\partial n_{0}}{\partial x}\right)+\Omega\left(n_{0}\right)
$$

where $\Phi, \Psi, \Omega$ are straightforward to obtain from (53).

While the constraint (47) of course holds for a wide range of $\sigma$ only in very special circumstances, it has the following (albeit somewhat fanciful) biological interpretation. In discrete systems of the type studied here, a continuum limit is applicable in those cases for which regimes of front pinning (propagation failure) are minimised - thus if patterning defects associated with pinning are undesirable, it would be advantageous for the biological system to fine-tune its kinetics in an attempt to satisfy (47). Conversely, if propagation failure is favoured, a strongly discrete regime would be appropriate (typically associated with weak coupling between cells).

\section{References}

Androutsellis-Theotokis, A., Leker, R., Soldner, F. , Hoeppner, D. , Ravin, R. , Poser, S. , Rueger, M. , Bae, S-K. , Kittappa, R. and McKay, R., 2006. Notch signalling regulates stem cell numbers in vitro and in vivo. Nat., 442:823-826. 
Appel, B. , Givan, L.A. and Eisen, J.S. , 2001. Delta-Notch signaling and lateral inhibition in zebrafish spinal cord development. BMC Dev. Biol, 1(13):1-13.

Burridge, R. and Keller, J.B. , 1981. Poroelasticity equations derived from microstructure. J. Acous. Soc. Am., 70:1140.

Campos-Ortega, J.A., 1993. Early neurogenesis in Drosophila melanogaster. Dev. Dros. Mel., 2:1091-1129.

Coffey, R.J., Derynck, R., Wilcox, J.N. , Bringman, T.S. , Goustin, A.S. , Moses, H.L. and Pittelkow, M.R., 1987. Production and auto-induction of transforming growth factor- $\alpha$ in human keratinocytes. Nature, 328(6133):817-820.

Collier, J.R. , Monk, N.A.M. , Maini, P.K. and Lewis, J.H. , 1996. Pattern Formation by Lateral Inhibition with Feedback: a Math. Model of Delta-Notch Intercellular Signalling. J. Theor. Biol., 183( 4):429-446.

Conboy, I. , Conboy, J. , Smythe, G. and Rando, T. , 2003. Notch-mediated restoration of regenerative potential to aged muscle. Sci., 302:1575-1577.

Dallon, JC and Othmer, HG , 1997. A discrete cell model with adaptive signalling for aggregation of Dictyostelium discoideum. Phil. Trans. of R. Soc. B: Biological Sciences, 352(1351):391.

Ebner, R and Derynck, R., 1991. Epidermal growth factor and transforming growth factor$\alpha$ : differential intracellular routing and processing of ligand-receptor complexes. Cell Reg., 2:599-612.

Fozard, J.A. , Byrne, H.M. , Jensen, O.E. and King, J.R. , 2009. Continuum approximations of individual-based models for epithelial monolayers. Math. Med. Biol. doi:10.1093/imammb/dri000.

Goel, P. , Sneyd, J. and Friedman, A. , 2006. Homogenization of the cell cytoplasm: the calcium bidomain equations. Multiscale Mod. \& Sim., 5:1045.

Haddon, C. , 1998. Delta-Notch signalling and the patterning of sensory cell differentiation in the zebrafish ear: evidence from the mind bomb mutant.

Hadeler, K.P. and Rothe, F. , 1975. Travelling fronts in nonlinear diffusion equations. J. Math. Biol., 2(3):251-263.

Hartenstein, V. and Posakony, J.W. , 1990. A dual function of the notch gene in drosophila sensillum development. Dev. Biol., 142(1):13-30.

Kevorkian, J. and Cole, J.D., 1996. Multiple scale and singular perturbation methods. Springer-Verlag, New York.

Lander, A.D. , Nie, Q. and Wan, F.Y.M. , 2002. Do morphogen gradients arise by diffusion? Dev. Cell, 2(6):785-796.

Lehmann, R., Jiménez, F. , Dietrich, U. and Campos-Ortega, J.A. , 1983. On the phenotype and development of mutants of early neurogenesis inDrosophila melanogaster. Dev. Genes and Evol., 192(2):62-74. 
Lowell, S. , Jones, P. , Le Roux, I. , Dunne, J. and Watt, F.M. , 2000. Stimulation of human epidermal differentiation by Delta-Notch signalling at the boundaries of stem-cell clusters. Curr. Biol., 10(9):491-500.

Mirams, G. , Byrne, H. and King, J. , 2010. A multiple timescale analysis of a mathematical model of the wnt/ $\beta$-catenin signalling pathway. J. Math. Biol., 60:131-160.

Mitsiadis, T.A. , Fried, K. and Goridis, C. , 1999. Reactivation of Delta-Notch signaling after injury: complementary expression patterns of ligand and receptor in dental pulp. Experimental Cell Res., 246(2):312-318.

Nusse, R. , 2008. Wnt signaling and stem cell control. Cell Res., 18:523-527.

Owen, M.R. , 2002. Waves and propagation failure in discrete space models with nonlinear coupling and feedback. Phys. D: Nonlin. Phenomena, 173(1-2):59-76.

Owen, M.R. and Sherratt, J.A., 1998. Mathematical modelling of juxtacrine cell signalling. Math. Biosci., 153(2):125-150.

Painter, K.J., Maini, P.K. and Othmer, H.G. , 1999. Stripe formation in juvenile Pomacanthus explained by a generalized Turing mechanism with chemotaxis. Proc. Nat. Acad. Sci. (US), 96(10):5549.

Plahte, E. , 2001. Pattern formation in discrete cell lattices. J. Math. Biol., 43(5):411-445.

Plahte, E. and Øyehaug, L. , 2007. Pattern-generating travelling waves in a discrete multicellular system with lateral inhibition. Phys. D: Nonlin. Phenomena, 226(2):117-128.

Rothe, F. , 1981. Convergence to pushed fronts. Rocky Mountain J. Math, 11(4):617-633.

Roussel, C.J. and Roussel, M.R. , 2004. Reaction-diffusion models of development with state-dependent chemical diffusion coefficients. Prog. in Biophys. and Molecular Biol., 86(1):113-160.

Shaapa, P. , Van Lookeren Campagne, M. , Van Driel, R. , Spek, W. , Van Haastert, P. and Pinas, J., 1986. Postaggregative differentiation induction by cyclic amp in dictyostelium: Intracellular transduction pathway and requirement for additional stimuli. Dev. Biol., 118:52-63.

Stokes, A.N. , 1976. On two types of moving front in quasilinear diffusion. Math. Biosci., 31(3-4):307-315.

Turing, A.M. , 1952. The Chemical Basis of Morphogenesis. Phil. Trans. Roy. Soc. Lond. Series B, Biol. Sci., 237(641):37-72.

Turner, S. , Sherratt, J.A. , Painter, K.J. and Savill, N.J. , 2004. From a discrete to a continuous model of biological cell movement. Phys. Rev. E, 69(2):21910/1-21910/10.

Webb, S.D. and Owen, M.R. , 2004a. Intra-membrane ligand diffusion and cell shape modulate juxtacrine patterning. J. Theor. Biol., 230(1):99-117.

Webb, S.D. and Owen, M.R. , 2004b. Oscillations and patterns in spatially discrete models for developmental intercellular signalling. J. Math. Biol., 48(4):444-476. 
Wiley, H. , Shvartsman, S. and Lauffenburger, D. , 2003. Computational modeling of the egf-receptor system: a paradigm for systems biology. Trends Cell Biol., 13:43-50.

Wolpert, L. , 1969. Positional information and the spatial pattern of cellular differentiation. J. Theor. Biol., 25(1):1-47. 